\title{
Nonholonomic Wheeled Mobile Robot Trajectory Tracking Control Based on Improved Sliding Mode Variable Structure
}

\author{
Hua Cen $\mathbb{D}^{1}$ and Bhupesh Kumar Singh $\mathbb{D}^{2}$ \\ ${ }^{1}$ Department of Mechanical and Electrical Engineering, Guangxi Modern Polytechnic College, Hechi 547000, China \\ ${ }^{2}$ Arba Minch Institute of Technology, Arba Minch University, Ethiopia
}

Correspondence should be addressed to Bhupesh Kumar Singh; dr.bhupeshkumarsingh@amu.edu.et

Received 28 April 2021; Accepted 1 June 2021; Published 18 June 2021

Academic Editor: VIMAL SHANMUGANATHAN

Copyright (C) 2021 Hua Cen and Bhupesh Kumar Singh. This is an open access article distributed under the Creative Commons Attribution License, which permits unrestricted use, distribution, and reproduction in any medium, provided the original work is properly cited.

\begin{abstract}
Several research studies are conducted based on the control of wheeled mobile robots. Nonholonomy constraints associated with wheeled mobile robots have encouraged the development of highly nonlinear control techniques. Nonholonomic wheeled mobile robot systems might be exposed to numerous payloads as per the application requirements. This can affect statically or dynamically the complete system mass, inertia, the location of the center of mass, and additional hardware constraints. Due to the nonholonomic and motion limited properties of wheeled mobile robots, the precision of trajectory tracking control is poor. The nonholonomic wheeled mobile robot tracking system is therefore being explored. The kinematic model and sliding mode control model are analyzed, and the trajectory tracking control of the robot is carried out using an enhanced variable structure based on sliding mode. The shear and sliding mode controls are designed, and the control stability is reviewed to control the trajectory of a nonholonomic wheeled mobile robot. The simulation outcomes show that the projected trajectory track control technique is able to improve the mobile robot's control, the error of a pose is small, and the linear velocity and angular speed can be controlled. Take the linear and angular velocity as the predicted trajectory.
\end{abstract}

\section{Introduction}

A mobile robot is a dynamic nonlinear device, with the benefits of unmanned driving and different controlling practices [1]. A wide range of applications covering survey, patrouille, emergency saving, recognition, and petrochemical features in automation industry, building structure, transport, health care, and other applications are being used by WMR from the last two decades. With the continuous improvement of robot performance, the application range of mobile robots is greatly expanded, and it is widely used in industry, agriculture, service industry, and other industries [2]. Therefore, it has become the hot spot of many scholars in recent years. Among them, wheeled mobile robot (WMR) has a wide range of practical application background [3]. This kind of system is a typical nonholonomic dynamic system because the rolling contact between the wheel and the ground must satisfy the nonholonomic constraint of pure rolling and no slipping. With the extensive feature of wheeled mobile robots
(WMR) in various fields, the precision requirements for the motion trajectory control of wheeled mobile robots are also higher and higher $[4,5]$. Each application has specific constraints to the robot design process, which is minimized if all compromises are interpreted by choosing the most convenient drive configuration.

Pappalardo and Guida [6] analyzed the dynamics and control method of nonholonomic robots, studied the Udwadia kalaba equation and underactuated equivalent principle, and carried out corresponding motion control for the robot. Taking the dynamic model of a mobile robot as an example, numerical tests conducted to verify the effectiveness of the projected control method. In addition to preventing distinctiveness nonetheless also keeping the benefits of the mode control sliding system, Zhao et al. [7] have suggested an uncompulsive wild nonsingular fatal sliding mode control for mobile robots. The experimental results show that the stability of the control method is high, and the tracking error of the robot trajectory is small. 
Ernesto et al. [2] suggested that the response be improved through kinematic model when the robot is backwardoriented and with a short distance to reach the location. Spandan et al. [8] did a novel stability study for the time-related control and gave an empirical relation between the output of the control and the approximation error. In the case where the wheel velocity is not existing deprived in regard to parametrical uncertainty, Shi et al. [9] stated the controller for the case. Bessas et al. [10] have developed an integrated mode controller based on the system's kinematic model. In consideration of several kinematic models, Onat and Ozkan [11] designed an adaptive parameter recognition controller. Based on the tracking error, the controller is moved to different kinematic models. In a Cartesian Coordinate, Lee et al. [12] developed the SMC approach angle. The risk of a switching effect due to high switching gains is a significant drawback of the switching control strategy, which can in turn lead to high frequency unmodelled dynamics affecting the robotic system. In addition, determining the uncertainty limits requires a nominal modelling of the system which, for a complex system such as WMR, is another tedious process [13].

The parametric uncertainties in the actuator were approximated by the Arab and Fateh [14] fuszy logic, while the core controller used the system's linearization approach to ignore system uncertainty. Application of response linearization-based control or converse dynamic control in real-life circumstances is not continually achievable because it needs a precise understanding of the hardware constraints. In view of the actuator limitation, Shojaei [15] recorded a strong controller with known uncertainty connection. Time-delayed control (TDC) [16] approximates all incertitude's using immediate past-instant control input and state information. TDC output however is influenced by the TDE that occurs when the time is finite.

A large number of application domains extending from surveillance, planetary exploration, patrolling, emergency rescue operations, reconnaissance, petrochemical applications, industrial automation, construction, entertainment, museum guides, personal services, intervention in extreme environments, transportation, medical care, and other industrial and nonindustrial applications are making use of wheeled mobile robots since the past two decades. Each application faces unique limitations on the robot design procedure which is reduced by selecting the most convenient drive configuration interpreting all the compromises.

The shear feature and sliding mode controller were established in accordance with the sliding mode control theory in accordance with the study of the mobile robot kinematic model. The control stability is studied to diminish the posing fault, and the trajectory of nonholonomic mobile robot control is carried out.

\section{Trajectory Tracking Control in accordance with Improved Sliding Mode Variable Structure}

The trajectory tracking problem of nonholonomic WMR is to propose a virtual mobile robot model which can perfectly track the ideal space trajectory and then let the actual robot track. Depending on the spatial coordinates, the desired movement of the nonholonomic robot can be expressed [6]. Different robot states, such as angular velocity, linear velocity, and spatial location, differ in time. The tracking trajectory not only included details about the spatial location but also the kinematic model and tracking trajectory feature of the nonholonomic mobile robot with wheels, as well as information on speeds and mobile angles [17]. As shown in Figure 1, the two driving wheels are autonomously determined through 2 DC motors. The follow-up wheel only plays a supporting role in the moving process, and its influence in the kinematic model can be ignored.

Figure 1 shows nonholonomic wheeled moving robot (WMR) powered by two engines attached to a radius $r$ at distance $b$ of the two wheels. The vehicle length is regarded as $L$. The $x$-axis of the axle of the robot in the center of mass is located by a moving body-fixed coordinate system, and the distance offset is supposed to apply. Let $P$ also stands for the WMR mass deprived of the driving wheels, rotor, and gearbox rotors; $w$ be each wheel's mass and the appropriate motor-rotor and gearbox rotor; $I_{w}$ stands for the wheel MOI equivalent to the wheel axis; and $I_{m}$ is the MOIs of each wheel with a rotor engine and gearbox that passes through the $z$-axis with a wheel diameter. Three generalized coordinates, $x_{c}, y_{c}$, and $\theta$, will specify the location of the WMR. The COM location here $\left(x_{c}, y_{c}\right)$ is the WMR heading angle, and $\theta$ is the WMR heading angle. Again, two more variables $\theta_{r}$ and $\theta_{l}$, indicating the rotation of the right-wing and leftwing wheels, are specified to identify some point on the wheels.

2.1. Nonholonomic Constraints for Wheeled Mobile Robots. As a characteristic nonholonomic control scheme, the motion of WMR is not only limited by the basic requirements of starting point and ending point but also meets the special requirements of velocity direction or trajectory shape. In addition, WMR is an essentially nonlinear under actuated drift-free dynamic system, which brings greater challenges to its control $[18,19]$. However, nonintegrity makes the structure of the robot more flexible and reliable, such as allowing some joints of the robot to be driveless, which greatly reduces the manufacturing cost, quality, and energy consumption of the robot. The nonholonomic control system represented by nonholonomic wheeled mobile robot is a new type of subject, which has important practical value and theoretical significance.

The motion behavior of the mobile robot is constrained by kinematics, which is determined by the structural characteristics of the robot and its motion model. In practical applications, the system is always subject to various constraints, such as geometric constraints and motion constraints, steady and unsteady constraints, double-sided constraints and single-sided constraints, integrable constraints, and nonintegrable constraints [20-23]. Under different constraints, the motion of the control system shows different characteristics. From the essence of system motion, constraints are divided into complete constraints and nonholonomic constraints. Nonholonomic constraints restrict the driving 


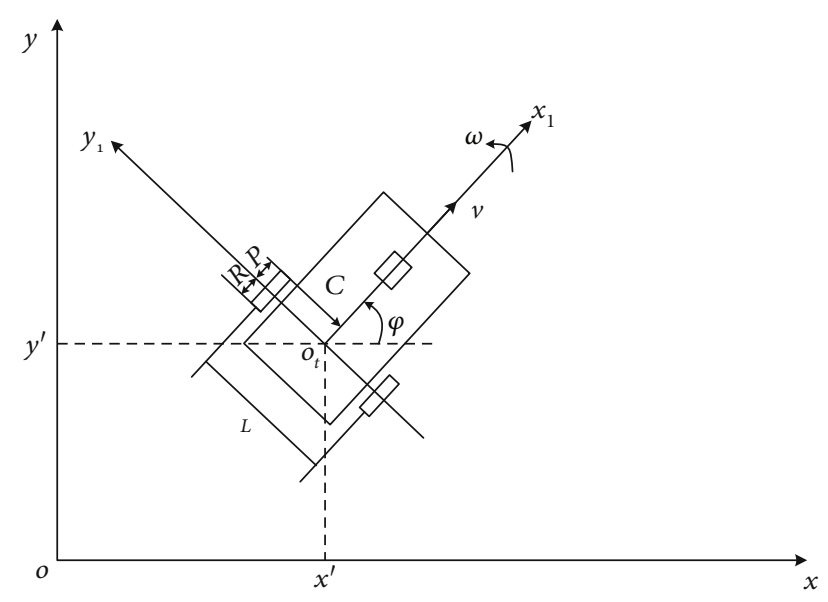

FIGURE 1: Representation of nonholonomic wheeled mobile robot.

direction of robots. Holonomic constraints are dimensions relative to the state description of the system. Suppose a constraint form:

$$
f(\mathbf{q}, \dot{\mathbf{q}}, t)=0
$$

where $\mathbf{q}=\left[q_{1}, q_{2}, \cdots, q_{n}\right]$ is the generated space vector. If this form of constraint can be transformed into a form:

$$
F(\mathbf{q}, t)=0
$$

then the constraint form is integrable. Even if $f$ in Equation (1) contains the differential quantity of generating space vector in time, it can be expressed by Equation (2), which is usually called complete constraint.

A nonholonomic constraint is defined as a time derivative including variables (coordinates) in the generating system and cannot be integrated. Nonholonomic constraint is a characteristic of the mechanical system. When the dimension of the control variable is lower than the state variable, the control variable can control the state variable [24, 25]. If the system has nonholonomic constraints, the system is nonholonomic, so it cannot move in any path (for example, the car will drive forward or backward, not side-by-side) [26]. The movement of this system is not arbitrary for nonholonomic systems; nonholonomic restrictions specify a subspace in the current time of the potential speed space. The return of internal variables to the initial value in a nonholonomic system does little to ensure that the system returns to the initial state. The condition of a nonholonomic system therefore depends on the route (a sequence set of internal variables that vary according to time). The coordinate dimension for the generated space is not reduced by nonholonomic restrictions excluding the generated speed space.

If the constraint of Equation (1) is integrable, its kinematic constraint is complete; that is, the velocity $q_{1}, q_{2}, \cdots$, $q_{n}$ can be eliminated from the equation. If the constraint of Equation (1) is not integrable, then the corresponding kinematic constraint is incomplete.
2.2. Kinematic Model Analysis. Mobile robots through nonholonomic components are called nonholonomic mobile robots. It belongs to the category of nonholonomic constraints, and its nonholonomic constraints mainly affect the motion characteristics of nonholonomic systems. The nonholonomic constraints received by wheeled mobile robots are generally expressed as first-order differential equations.

In the plane, the position and attitude of the robot can be represented by the following state vectors:

$$
\mathbf{x}_{m}(t)=\left[\begin{array}{c}
x_{m}(t) \\
y_{m}(t) \\
\theta_{m}(t)
\end{array}\right]
$$

$\left(x_{m}, y_{m}\right)$ represents the position of robot global coordinate system, and $\theta_{m}$ represents the steering angle of robot global coordinate system. $\mathbf{x}_{m}$ represents the pose state of the robot at time $t$.

To keep the balance in the plane, this type of robot usually has one or more castors as additional support only. The driving control is completed by motors which are separately distributed in two wheels. In the universal organize scheme, the motion equation of the robot can be formulated as:

$$
\left[\begin{array}{l}
\dot{x}_{m}(t) \\
\dot{y}_{m}(t) \\
\dot{\theta}_{m}(t)
\end{array}\right]=\left[\begin{array}{cc}
\cos \theta_{m}(t) & 0 \\
\sin \theta_{m}(t) & 0 \\
0 & 1
\end{array}\right]\left[\begin{array}{c}
v(t) \\
w(t)
\end{array}\right] \text {. }
$$

Its nonholonomic motion constraints are as follows:

$$
-\dot{x}_{m} \sin \theta_{m}+\dot{y}_{m} \cos \theta_{m}=0 \text {. }
$$

Equation (5) can be written in terms of the direction of motion that can be realized by the motion matrix

$$
S=\left[\begin{array}{cc}
\cos \theta_{m}(t) & 0 \\
\cos \theta_{m}(t) & 0 \\
0 & 1
\end{array}\right] \text {. }
$$

$v(t)$ and $w(t)$ are usually regarded as control variables in robot motion research. After applying the Euler integral to Equation (4), it can be written as follows:

$$
\begin{gathered}
x_{m}(k+1)=x_{m}(k)+v(k) \Delta t \cos \left(\theta_{m}(t)\right), \\
y_{m}(k+1)=y_{m}(k)+v(k) \Delta t \sin \left(\theta_{m}(t)\right), \\
\theta_{m}(k+1)=\theta_{m}(k)+w(k) \Delta t,
\end{gathered}
$$

where $k$ is the discrete time constant and $\Delta t$ is the time interval.

There are many ways to make the robot move to the ideal position by using the plane changing trajectory. The inverse kinematic challenge is easier for the robot to plane the target trajectory $(x(t), y(t))$, so that the direction of the robot is always tangent to the trajectory. If the initial pose of the 
reference robot is on a certain trajectory, then there is a kinematic model corresponding to the physical quantity of the reference robot. It can be concluded that the control linear velocity is $v(t)$ :

$$
v(t)= \pm \sqrt{\dot{x}^{2}(t)+\dot{y}^{2}(t)}
$$

The positive and negative signs represent the ideal driving direction ( + is the forward direction, and - is the reverse direction). Every point on the track has a tangent angle defined as follows:

$$
\theta(t)=\arctan 2(\dot{y}(t), \dot{x}(t))+k \pi,
$$

where $k \in\{0,1\}$ defines the ideal driving direction ( 0 is the forward direction, and 1 is the reverse direction). By calculating the differential of time in Equation (9), the angular velocity of the robot can be obtained:

$$
w(t)=\frac{\dot{x}(t) \ddot{y}(t)-\dot{y}(t) \ddot{x}(t)}{\dot{x}^{2}(t)+\dot{y}^{2}(t)} .
$$

By using the Equations (8) and (9), the ideal trajectory control is obtained.

2.3. Sliding Mode Control Principle. When the system does not satisfy the linear parameterization condition or when the system model has both structural and parametric uncertainties, a sliding mode control algorithm can be used. If the upper bound function can be found for the unknown dynamic characteristics, the sliding mode control algorithm can achieve the desired control effect by strengthening the control input, that is, using very strong control variables to deal with the unknown dynamic characteristics. Sliding mode control has strong control performance for nonlinear systems affected by uncertain dynamics, which makes the system move according to the designed sliding mode trajectory. The control method has the advantages of a simple calculation method and strong anti-interference ability.

Sliding mode control for nonlinear systems influenced by unknown factors is an easy and efficient way of control. The problem can be reduced to a complex one by simple sliding mode control. The nonlinear method for the single input output can be expressed as follows:

$$
y^{(n)}=f(\bar{y})+u
$$

$u \in R$ and $y \in R$ are control input variables and system output variables, respectively, and $\bar{y}=\left[y, \dot{y}, \cdots, y^{(n-1)}\right]^{T} \in R^{n}$ is system state vector.

In order to make the trajectory tracking error $\bar{e}=\bar{y}-\bar{y}_{d}$ $=\left[e, \dot{e}, \cdots e^{(n-1)}\right]^{1}$ of the controlled system converge to zero, that is, the state variables of the closed-loop system can gradually track the reference trajectory $\bar{y}_{d}=\left[y_{d}, \dot{y}_{d}, \cdots y_{d}^{(n-1)}\right]^{T}$, a state feedback control law needs to be designed by using sliding mode controller. The PI sliding mode variable structure controller is defined as follows:

$$
s(y, t)=e(t)+\gamma \int_{0}^{t} e(\tau) d \tau .
$$

Among them, $\gamma>0$. When $s(y, t)=0$ is set, $e=0$ is the only solution to Equation (12). Then, the problem of tracking the desired trajectory $y_{d}$ is equivalent to the problem of function $s(y, t)=0$. At the same time, the selection of control law $u$ should satisfy the following conditions:

$$
\frac{1}{2} \frac{d}{d t} s^{2} \leq-\rho|s|
$$

where $\rho>0$ is a constant. Equation (13) is called sliding condition, which guarantees that the error state can converge from the initial position far away from $s(y, t)=0$ surface to $s(y, t)=0$ surface.

The strategy of sliding mode controller mainly comprises 2 parts: the equivalent control variable $u_{\mathrm{eq}}$ is designed for the ideal sliding mode control, and the variable structure control variable $u_{\text {sw }}$ is also designed for the nonlinear system affected by various uncertain factors. The design of sliding mode variable structure controller consists of two steps:

Step 1. Design $s$ switching function to make sliding mode stable and dynamic.

Step 2. The sliding mode control law $u$ is designed to form a sliding mode region on the switching surface $s(y, t)=0$ under the condition that the condition Equation (13) is satisfied [27].

For uncertain systems, using the sliding mode variable structure control method can often get the desired results. Compared with the traditional control algorithm, the sliding mode production variable structure control method is discrete; that means, the high-frequency switch control is used to switch between several control laws, which can make the system oscillate with small amplitude and high frequency near the set space surface. Such motion is defined as sliding mode motion. Since various control laws in the variable control method for sliding mode are set in advance and are not disturbed and do not rely on device parameters, this method is highly robust. Then, the concept of sliding mode control combines a trajectory tracking controller with global asymptotic stability.

\subsection{Design Shear Function}

Lemma 1. Any $x \in R$ and $|x|<\infty$, then $\varphi(x)=x \sin (\arctan$ $x) \geq 0$, if and only if $x=0$, the equal sign holds. The switching function of sliding mode variable structure control is designed based on backstepping method; firstly, the Lyapunov function is defined as $V_{y}=(1 / 2) y_{e}^{2}$; suppose $x_{e}=c f(k \omega) y_{e}$, where $f(k$ $\omega)=(1-\exp (-2 k \omega)) /(1+\exp (-2 k \omega))$ is a hyperbolic tangent function and $c f(k \omega) y_{e}$ is a virtual feedback variable; $c$ and $k$ were normal. According to the characteristics of 
hyperbolic tangent function, we can know $\omega f(k \omega) \geq 0$. Assuming $\phi_{e}=-\arctan \left(u_{r} y_{e}\right)$, then

$$
\dot{\phi}=\frac{\partial \phi_{e}}{\partial u_{r}}+\frac{\partial \phi_{e}}{\partial y_{e}}=\frac{u_{r}+y_{e}}{1+\left(u_{r} y_{e}\right)^{2}}<0
$$

Based on Equation (14) and Lemma 1, the following equation can be given

$$
\begin{aligned}
\dot{V}_{y}= & y_{e} \dot{y}_{e}=y_{e}\left(-x_{e} \omega-a \omega+u_{r} \sin \varphi_{e}+a \omega_{r} \cos \varphi_{e}\right) \\
= & -y_{e} x_{e} \omega-y_{e} a \omega+u_{r} y_{e} \sin \left(-\arctan \left(u_{r} y_{e}\right)\right) \\
& +y_{e} a \omega_{r} \cos \left(-\arctan \left(u_{r} y_{e}\right)\right) \leq-y_{e} x_{e} \omega-y_{e} a \omega \\
& +y_{e} a \omega_{r}=-y_{e}^{2} c f(k \omega) \omega+y_{e} a \dot{\varphi}_{e}<0 .
\end{aligned}
$$

It is concluded that as long as $x_{e}$ converges to $c f(k \omega) y_{e}$ and $\varphi_{e}$ converges to $-\arctan \left(u_{r} y_{e}\right)$, the system state variable $y_{e}$ will converge to 0 . According to this conclusion, the switching function is designed as follows:

$$
s=\left[\begin{array}{l}
s_{1} \\
s_{2}
\end{array}\right]=\left[\begin{array}{c}
x_{e}-c f(k \omega) y_{e} \\
\varphi_{e}+\arctan \left(u_{r} y_{e}\right)
\end{array}\right] .
$$

Through the design of sliding mode controller, $s_{1}$ and $s_{2}$ tend to 0 , which means $x_{e}$ converges to $c f(k \omega) y_{e}, \varphi_{e}$ converges to 0 , so the system state variable $y_{e}$ also converges to 0 .
2.5. Design of Sliding Mode Controller. To weaken the jitter, the exponential reaching law $\dot{s}=-\varepsilon$ sgn $s-h s$ is adopted, in which $\varepsilon$ and $h$ are greater than 0 , and $\varepsilon$ is a small positive number. The following equation can be obtained by using $S$ type continuous function $\left(1-e^{-s}\right) /\left(1+e^{-s}\right)$ instead of symbol function:

$$
\dot{s}_{i}=-\varepsilon_{i} \frac{1-e^{-s_{i}}}{1+e^{-s_{i}}}-h_{i} s_{i}, \quad i=1,2 .
$$

$\varepsilon_{i}$ is a small positive number, and $h_{i}$ is a normal number. Suppose $\alpha=\arctan \left(u_{r} y_{e}\right)$, from Equation (16) and Equation (17), we can get the following results:

$$
\begin{aligned}
\dot{s}_{i} & =\left[\begin{array}{l}
\dot{s}_{1} \\
\dot{s}_{2}
\end{array}\right]=\left[\begin{array}{c}
-\varepsilon_{1} \frac{1-e^{-s_{1}}}{1+e^{-s_{1}}}-h_{1} s_{1} \\
-\varepsilon_{2} \frac{1-e^{-s_{2}}}{1+e^{-s_{2}}}-h_{2} s_{2}
\end{array}\right]=\left[\begin{array}{c}
\dot{x}-c k f^{\prime}(k \omega) \dot{\omega} y_{e}-c f(k \omega) \dot{y}_{e} \\
\dot{\phi}_{e}+\frac{\partial \alpha}{\partial u_{r}} \dot{u}_{r}+\frac{\partial \alpha}{\partial y_{e}} \dot{y}_{e}
\end{array}\right] \\
& =\left[\begin{array}{c}
y_{e} \omega-u+u_{r} \cos \varphi_{e}-\mathrm{a} \omega_{r} \sin \varphi_{e}-c k f^{\prime}(k \omega) \dot{\omega} y_{e}-c f(k \omega) \dot{y}_{e} \\
\omega_{r}-\omega+\frac{\partial \alpha}{\partial u_{r}} \dot{u}_{r}+\frac{\partial \alpha}{\partial y_{e}} \dot{y}_{e}
\end{array}\right] .
\end{aligned}
$$

From Equation (18), the kinematic trajectory tracking control law of the nonholonomic wheeled mobile robot with discrepancy drive can be obtained

$$
\left[\begin{array}{c}
u \\
\omega
\end{array}\right]=\left[\begin{array}{c}
y_{e}+u_{r} \cos \varphi_{e}-a \omega_{r} \sin \varphi_{e}-c k f^{\prime}(k \omega) \dot{\omega} y_{e}-c f(k \omega) \dot{y}_{e}+\varepsilon_{1} \frac{1-e^{-s_{1}}}{1+e^{-s_{1}}}-h_{1} s_{1} \\
\frac{\omega_{r}+\left(\partial \alpha / \partial u_{r}\right) \dot{u}_{r}+\left(\partial \alpha / \partial y_{e}\right)\left(u_{r} \sin \varphi_{e}+\mathrm{a} \omega_{r} \cos \varphi_{e}\right)+\varepsilon_{2}\left(\left(1-e^{-s_{2}}\right) /\left(1+e^{-s_{2}}\right)\right)-h_{2} s_{2}}{1+\left(\partial a / \partial_{y_{e}}\right)\left(x_{e}+a\right)}
\end{array}\right] .
$$

When $a=0$ is chosen, the trajectory tracking control law of discrepancy drive nonholonomic wheeled mobile robot is as follows

$$
\begin{gathered}
{\left[\begin{array}{c}
u_{0} \\
\omega_{0}
\end{array}\right]=\left[\begin{array}{c}
y_{e} \omega+u_{r} \cos \varphi_{e}-c k f^{\prime}(k \omega) \dot{\omega} y_{e}-c f(k \omega) \dot{y}_{e}+\varepsilon_{1} \frac{1-e^{-s_{1}}}{1+e^{-s_{1}}}-h_{1} s_{1} \\
\frac{\omega_{r}+\left(\partial \alpha / \partial u_{r}\right) \dot{u}_{r}+\left(\partial \alpha / \partial y_{e}\right)\left(u_{r} \sin \varphi_{e}\right)+\varepsilon_{2}\left(\left(1-e^{-s_{2}}\right) /\left(1+e^{-s_{2}}\right)\right)-h_{2} s_{2}}{1+\left(\partial a / \partial_{y_{e}}\right) x_{e}}
\end{array}\right],} \\
\frac{\partial \alpha}{\partial u_{r}}=\frac{y_{e}}{1+\left(u_{r} y_{e}\right)^{2}}, \frac{\partial \alpha}{\partial y_{e}}=\frac{u_{r}}{1+\left(u_{r} y_{e}\right)^{2}} .
\end{gathered}
$$

2.6. Stability Analysis. Because the sliding mode variable structure control requires that the state quantity of the motion on the sliding surface must be the end point, when the moving point reaches the sliding surface, it must meet the following requirements: $\lim _{s \rightarrow 0} \dot{s} \leq 0$. The following Lyapu- nov function is defined as $[28,29]$

$$
V=\frac{1}{2} s_{1}^{2}+\frac{1}{2} s_{2}^{2}
$$


By deriving Equation (21) and substituting it into Equation (17), we can get the following results:

$$
\dot{V}=-h_{1} s_{1}^{2}-h_{2} s_{2}^{2}-\varepsilon_{1} s_{1} \frac{1-e^{-s_{1}}}{1+e^{-s_{1}}}-\varepsilon_{2} s_{2} \frac{1-e^{-s_{2}}}{1+e^{-s_{2}}}
$$

Because $\dot{V} \leq 0$, so $V$ is a monotone nonincreasing function; $V_{t \leq} V_{0}, V_{t}$ is bounded on $t \in[0, \infty)$; therefore, from Equations (16) and (21), $x_{e}, y_{e}, \varphi_{e}$, and $\omega$ are bounded values; it is also known from Equation (19) that $\dot{\omega}$ expected linear velocity $u_{r}$ and angular velocity $\omega_{r}$ are bounded quantities; from error Equation (13), $\dot{x}_{e}, \dot{y}_{e}$, and $\dot{\varphi}_{e}$ are bounded; that is, $x_{e}, y_{e}$, and $\varphi_{e}$ are consistent continuous variables; according to Barbarat theorem, $x_{e}, y_{e}$, and $\varphi_{e}$ converge to zero when $t \longrightarrow \infty$.

Theorem 2. The Lyapunov function candidate be $V(\mathbf{e})=\mathbf{e}^{T}$ Me, where $\mathbf{M}$ is the result of the Lyapunov equivalence. The stability aspects of the nonholonomic WMR are considered for diverse cases represented as follows:

Case 1. When $\|s\| \geq €$.

The time derivative of $V$ gives,

$$
\begin{aligned}
V^{\prime}(\mathbf{e})= & \mathbf{e}^{T} \mathbf{M e}+\mathbf{e}^{T} \mathbf{M} \mathbf{e}^{\prime}=e^{T}\left(\mathbf{A}^{T} \mathbf{M}+\mathbf{M A}\right) \mathbf{e} \\
& +2 \mathbf{e}^{T} \mathbf{M B}\left(\boldsymbol{\Delta} \mathbf{u}_{\mathbf{a}+} \boldsymbol{\delta}\right)=-e^{T} N e+2 \mathbf{s}^{T}\left(-k \frac{s}{\|s\|}+\boldsymbol{\delta}\right) .
\end{aligned}
$$

Additional universalization of $\mathbf{V}^{\prime}(\mathbf{e})$ produces:

$$
\begin{aligned}
V^{\prime}(\mathbf{e}) & \leq-\mathbf{e}^{T} \mathbf{N e}-\mathbf{2} \mathbf{k}^{\prime}\|\mathbf{S}\|+2\|\mathbf{S}\| \mathbf{k} \\
& \leq-\lambda_{\min }(\mathbf{N})\|\mathbf{e}\|^{2}+2\left(\mathbf{k}-\mathbf{k}^{\prime}\right)\|\mathbf{S}\| \\
& \leq-\|\mathbf{e}\|\left(\lambda_{\min }(\mathbf{N})\|\mathbf{e}\|-2\left(\mathbf{k}-\mathbf{k}^{\prime}\right)\left\|\mathbf{B}^{\mathrm{T}} \mathbf{M}\right\|\right)
\end{aligned}
$$

So, ' $\mathbf{V}(\mathbf{e})<0$ may be conventional if $\|\mathbf{e}\|>\left(\mathbf{2}\left(\mathbf{k}-\mathbf{k}^{\prime}\right) \| \mathbf{B}\right.$ $\mathbf{T M} \|) / \boldsymbol{\lambda}_{\min }(\mathbf{N}):=\boldsymbol{\omega} \mathbf{2}$. Consequently, the structure may be ultimate bound through subsequent:

$$
\|\mathbf{e}\| \leq \frac{\sqrt{\left(\lambda \operatorname{mix}(\mathbf{M}) \omega^{2}\right.}}{\lambda \min (\mathbf{M})}
$$

The time to range $\omega 2$ can initiate as $\mathbf{t}_{r 2}-\mathbf{t}_{0} \leq\left(\mathbf{V}\left(\mathbf{e}\left(\mathbf{t}_{0}\right)\right)\right.$ $-\mathbf{V}(\boldsymbol{\omega} \mathbf{2})) / \mathbf{c}_{0}$.

Case 2. When $\|\mathbf{S}\|<€$.

The term $2 \mathbf{S}^{T} \delta$ may show as follows:

$$
2 \mathbf{s}^{T} \delta \leq \mathbf{2}\|\mathbf{S}\|\|\delta\| \leq \mathbf{2 k}\|\mathbf{S}\|=\mathbf{2 k}\left(\frac{\mathbf{S}^{T} S}{\|S\|}\right)
$$

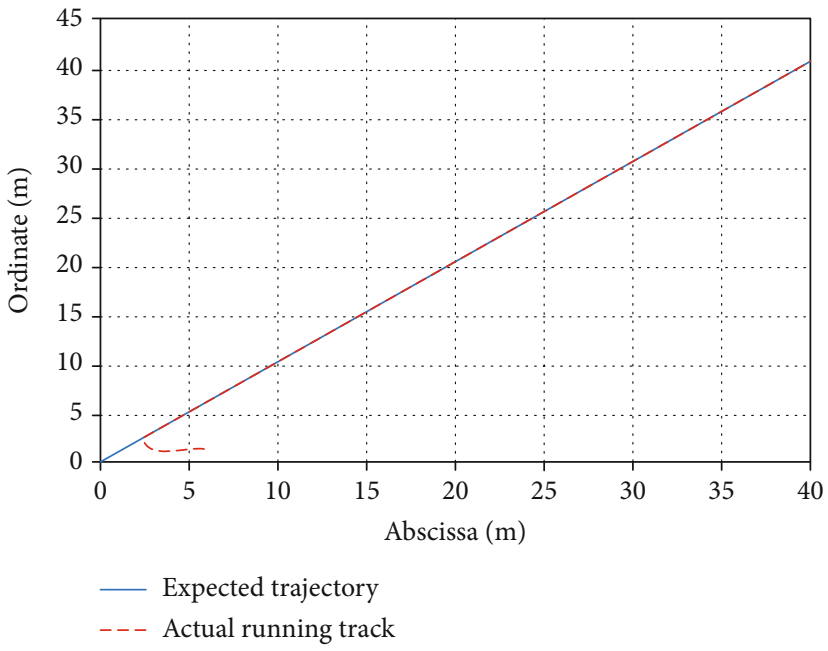

Figure 2: Track tracking effect.

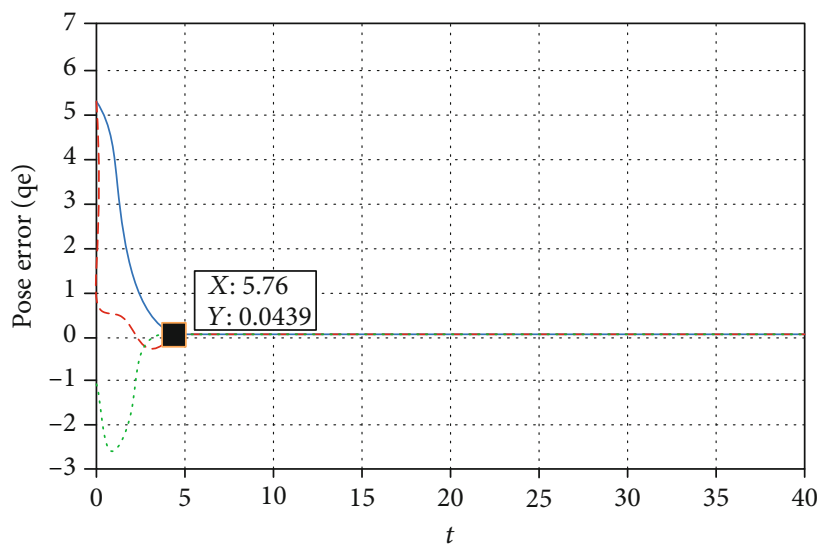

- Abscissa error/e1 (m)

- - - Ordinate error/e2 (m)

Angle error/e3 (rad)

Figure 3: Change curve of pose error.

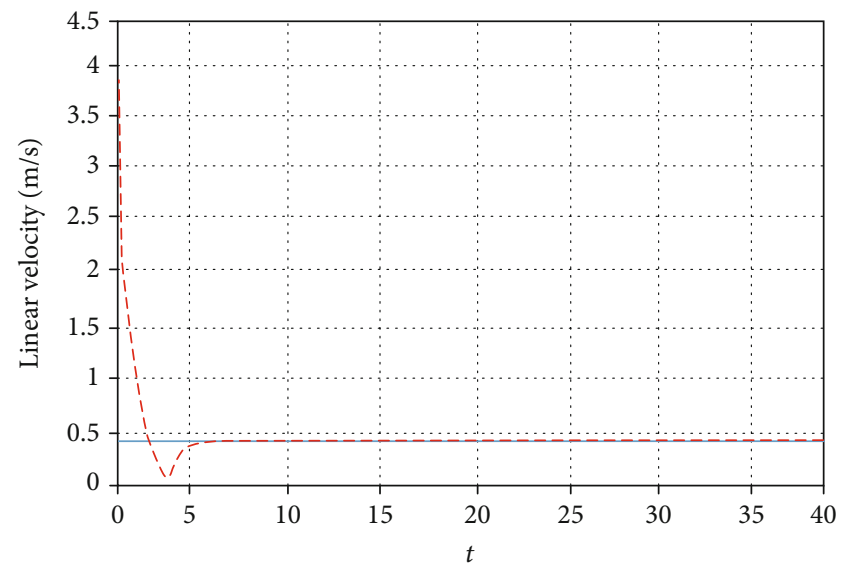

— Expected linear velocity $/ v r$

- - - Actual linear velocity $/ v$

FIGURE 4: Linear velocity variation curve. 


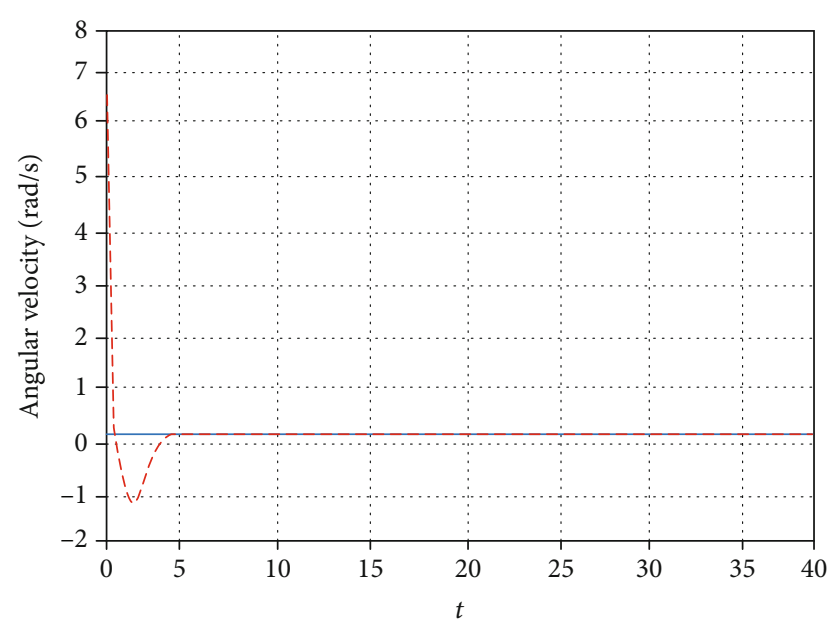

- Desired angular velocity/wr

- - Actual angular velocity $/ w$

FIgURE 5: Variation curve of angular velocity.

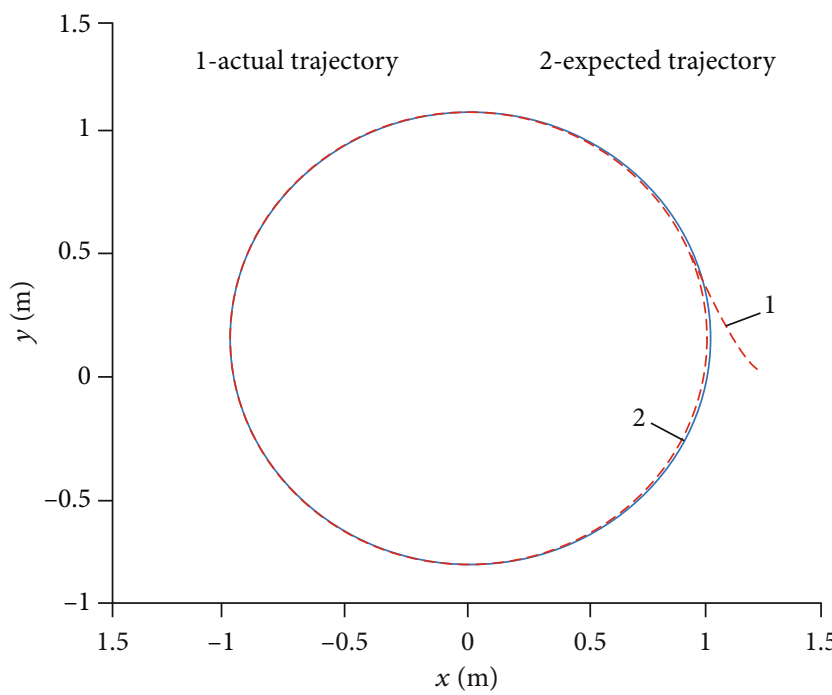

FIgURE 6: Track tracking effect.

By Equations (23) and (26), time directive $\mathbf{V}(\mathbf{e})$ produces

$$
\begin{aligned}
\mathbf{V}(\mathbf{e}) & \leq-\mathbf{e}^{T} \mathbf{N e}+2 \mathbf{s}^{\mathbf{T}}\left(\Delta \mathbf{u}_{\mathbf{a}}+\mathbf{k} \frac{\mathbf{S}}{\|\mathbf{S}\|}\right) \\
& \leq-\lambda_{\min }(\mathbf{N})\|\mathbf{e}\|^{2}+2 \mathbf{s}^{\mathbf{T}}\left(-\mathbf{k} \frac{\mathbf{S}}{€}+\mathbf{k} \frac{\mathbf{S}}{\|\mathbf{S}\|}\right)
\end{aligned}
$$

Equation (27) computes more value $\left(€ \mathbf{k}^{\mathbf{2}}\right) /\left(\mathbf{2} \mathbf{k}^{\prime}\right)$ when $\|\mathbf{S}\|=(\boldsymbol{\epsilon} \mathbf{k}) /\left(\mathbf{2} \mathbf{k}^{\prime}\right)$, so

$$
\left.\mathbf{V}(\mathbf{e}) \leq-\lambda_{\min }(\mathbf{N})\|\mathbf{e}\|^{2}+\frac{€ k 2}{2 k}\right)
$$

Yet over, $\mathbf{V}(\mathbf{e})<0$ may conventional if $\|\mathbf{e}\|>$

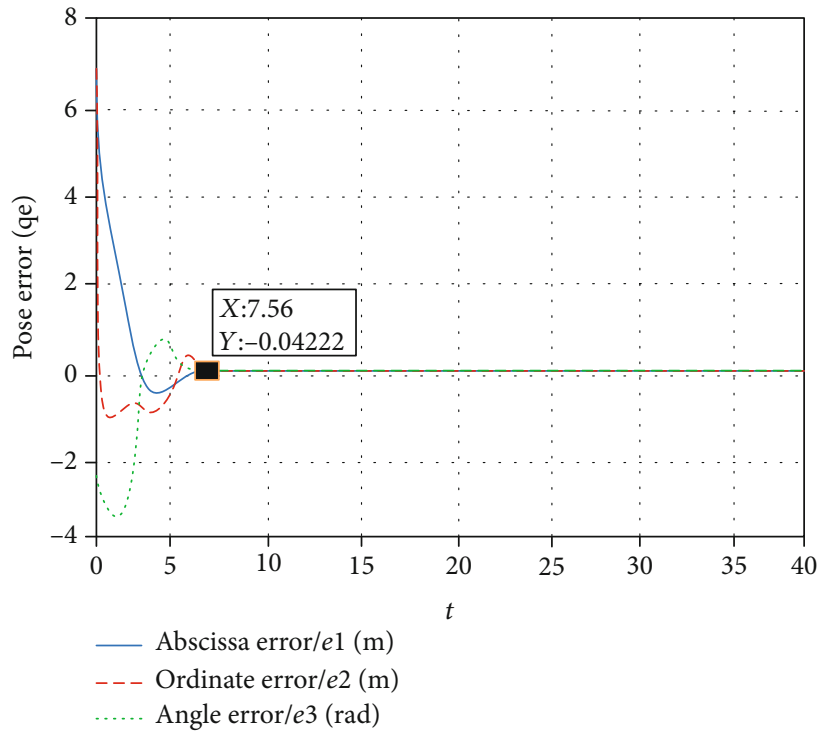

FIgURE 7: Change curve of pose error.

$\sqrt{(\boldsymbol{\epsilon} \mathbf{k} 2) /\left(\mathbf{2} \mathbf{k}^{\prime} \boldsymbol{\lambda} \min (\mathbf{Q})\right)}:=\boldsymbol{\omega} \mathbf{3}$. Consequently, the structure is ultimate bound with the subsequent:

$$
\|\mathbf{e}\| \leq \sqrt{\frac{\left.\lambda \max (\mathbf{M}) \omega^{\prime} \mathbf{3}\right)}{\lambda \max (\mathbf{M})}} .
$$

Correspondingly, the time to influence $\omega 3$ can initiate as $\left.t_{r 3}-t_{0} \leq V\left(e\left(t_{0}\right)\right)-V(\dot{\omega} 3)\right) / c_{0}$.

Equations (23), (25), and (29) can easily be understood as $\omega 3<\omega 2<\omega 1$. In addition, $€$ is chosen very small in practice, and thus, $\dot{\omega} 3$ can be minimized by correct tuning of $€$ and $N$ additionally.

\section{Simulation Experiment Verification}

Considering that line and circle are common expected trajectory forms in practical application, this paper selects linear and circular trajectories to simulate and verify the above tracking control law and carries out the corresponding trajectory tracking controller simulation for nonholonomic WMR. The excessive acceleration caused by excessive speed will destroy the nonholonomic constraints of the robot, which is easy to cause the lateral movement or longitudinal sliding of the mobile robot. To avoid the above problems, the speed of the mobile robot must be limited. The maximum speed is $1.5 \mathrm{~m} / \mathrm{s}$, and the extreme angular velocity is $1.5 \mathrm{rad} / \mathrm{s}$. The experiments of WMR are considered as follows: the hardware components for the robotic stages are nominated as $m=34$ $\mathrm{kg}, d=0.14 \mathrm{~m}, r=0.085 \mathrm{~m}, b=0.504 \mathrm{~m}, I=4.89 \mathrm{~kg} \mathrm{~m}^{2}, I w$ $=0.08 \mathrm{~kg} \mathrm{~m}^{2}, k=0.36 \mathrm{~kg} \mathrm{~m}^{2}, I=6.13 \mathrm{~kg} \mathrm{~m}^{2}, I w=0.08 \mathrm{~kg} \mathrm{~m}^{2}$, and $K=0.33 \mathrm{kgm}$. Due to the communiqué interruption among the robot and control station and the related sensor suite and hardware, the sampling time $h$ is considered judiciously $100 \mathrm{~ms}$. In experimentation with wheel location and velocity, the wheel encoders (incremental encoder of square 


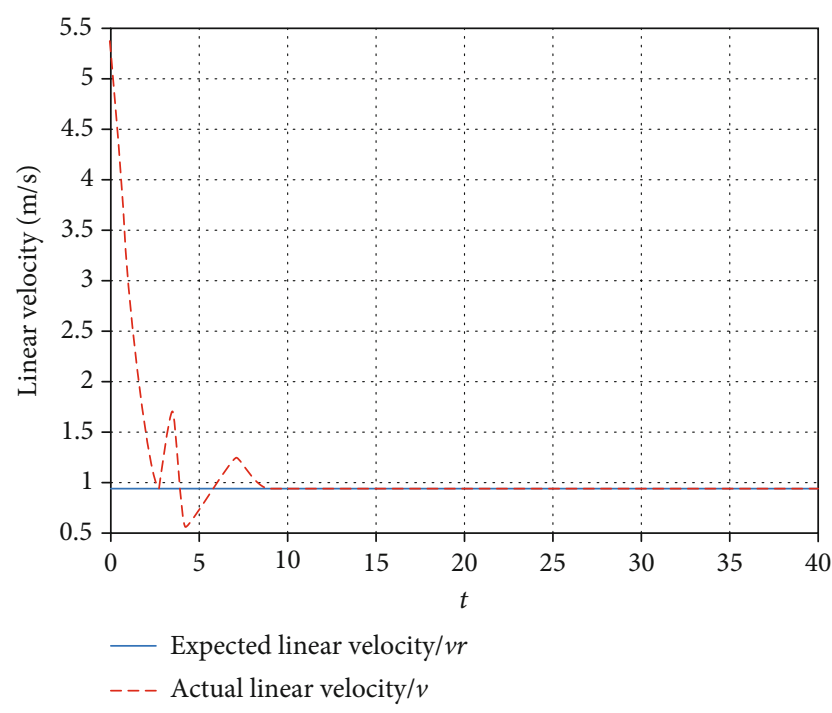

FIGURE 8: Linear velocity variation curve.

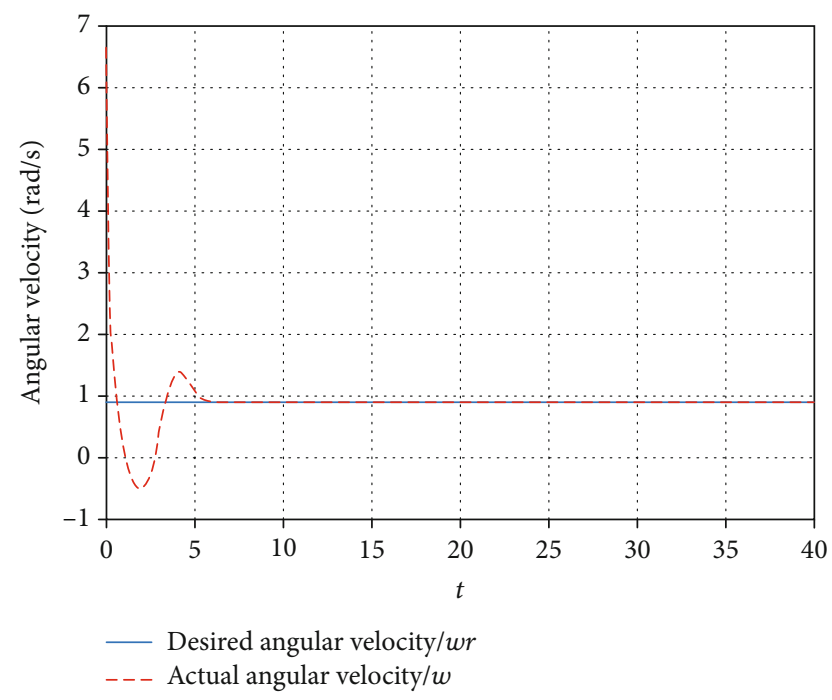

FIGURE 9: Variation curve of angular velocity.

design with $500 \mathrm{ppr}$ ) are used. Moreover, when the wheel velocities $\theta_{r}$ and $\theta_{l}$ are achieved, the following kinematic relationships integrate the remaining state variables.

3.1. Straight Track. When the trajectory is a straight line, the control input of the mobile robot is selected as follows: the primary linear velocity is $0.4 \mathrm{~m} / \mathrm{s}$, the angular velocity is 0.3 $\mathrm{rad} / \mathrm{s}$, and the controller parameters are set to 1.5 . The simulation results are shown in Figures 2-5.

It can be seen from Figure 2 that under the action of the control law, the nonholonomic WMR system can realize the straight-line trajectory tracking at the abscissa of about $5 \mathrm{~m}$ and finally converge to the given linear reference trajectory. The pose error can eventually converge to 0 and can be seen in Figure 3. The nonholonomic wheeled mobile robot device will, as shown in Figures 4 and 5, converge the anticipated linear velocity and the expected angular velocity under the control rule, which checks the effectiveness of the trajectory tracking process.

3.2. Circular Trajectory. When the trajectory is circular, the control input of the mobile robot is selected as follows: the primary linear velocity is $1 \mathrm{~m} / \mathrm{s}$, the angular velocity is $1 \mathrm{rad} / \mathrm{s}$, and the controller parameters are set to 1. Figures 6-9 show the results of the simulation.

From Figures 6-9 that under the action of the designed control law, the circular motion trajectory always has a good fit with the expected trajectory, and the pose error can eventually converge to 0 , and the linear velocity and angular velocity can also converge to the expected situation after different times. Therefore, the efficiency of the control law in tracking the circular trajectory of the nonholonomic WMR is verified.

In summary, it can be seen from the results of the above simulation of two tracking types on a nonholonomic mobile robot that the nonholonomic mobile robot system can progressively pursue the reference trajectory under the effect of a designed trajectory tracking law, the position error can converge to zero quickly, and the input of the speed control can converge also to the referee quickly. The efficacy of the proposed law on control is verified.

\section{Conclusion}

In this paper, the nonholonomic WMR trajectory control system based on improved variable structure of sliding mode is projected. Based on analyzing the principle of sliding mode control, the improved method is designed by adding stability analysis to reduce the pose error of trajectory tracking control. The experimental results verify the high control precision effect of the designed method and provide a basic theoretical reference for practical application.

\section{Data Availability}

Data are available on request

\section{Conflicts of Interest}

The authors declare no conflicts of interest.

\section{References}

[1] Y. Qiu, B. Li, W. Shi, and X. Zhang, "Visual servo tracking of wheeled mobile robots with unknown extrinsic parameters," IEEE Transactions on Industrial Electronics, vol. 66, no. 11, pp. 8600-8609, 2019.

[2] E. Fabregas, G. Farias, E. E. Aranda et al., "Simulation and experimental results of a new control strategy for point stabilization of nonholonomic mobile robots," IEEE Transactions on Industrial Electronics, vol. 67, no. 8, pp. 6679-6687, 2020.

[3] C. Della Santina, R. K. Katzschmann, A. Bicchi, and D. Rus, "Model-based dynamic feedback control of a planar soft robot: trajectory tracking and interaction with the environment," The International Journal of Robotics Research, vol. 39, no. 4, pp. $490-513,2020$. 
[4] D. Saravanakumar, A. Thomas, and J. Jarvis, "Bacillus strains in the control of bacterial wilt in tomato," Phytopathology, vol. 108, no. 10, p. 143, 2018.

[5] Y. Wang, M. Shan, Y. Yue, and D. Wang, "Vision-based flexible leader-follower formation tracking of multiple nonholonomic mobile robots in unknown obstacle environments," IEEE Transactions on Control Systems Technology, vol. 28, no. 3, pp. 1025-1033, 2020.

[6] C. M. Pappalardo and D. Guida, "On the dynamics and control of underactuated nonholonomic mechanical systems and applications to mobile robots," Archive of Applied Mechanics, vol. 89, no. 4, pp. 669-698, 2019.

[7] J. Y. Zhai and Z. B. Song, "Adaptive sliding mode trajectory tracking control for wheeled mobile robots," International Journal of Control, vol. 92, no. 10, pp. 1-16, 2018.

[8] S. Roy, S. Nandy, I. N. Kar, R. Ray, and S. N. Shome, "Robust control of nonholonomic wheeled mobile robot with past information: theory and experiment," Proceedings of the Institution of Mechanical Engineers Part I Journal of Systems and Control Engineering, vol. 231, no. 3, pp. 178-188, 2017.

[9] S. Shi, X. Yu, and S. Khoo, "Robust finite-time tracking control of nonholonomic mobile robots without velocity measurements," International Journal of Control, vol. 89, no. 2, pp. 411-423, 2016.

[10] A. Bessas, A. Benalia, and F. Boudjema, "Integral sliding mode control for trajectory tracking of wheeled mobile robot in presence of uncertainties," Journal of Control Science and Engineering, vol. 2016, Article ID 7915375, 10 pages, 2016.

[11] A. Onat and M. Ozkan, "Dynamic adaptive trajectory tracking control of nonholonomic mobile robots using multiple models approach," Advanced Robotics, vol. 29, no. 14, pp. 913-928, 2015.

[12] J. K. Lee, Y. H. Choi, and J. B. Park, "Sliding mode tracking control of mobile robots with approach angle in Cartesian coordinates," International Journal of Control, Automation, vol. 13, no. 3, pp. 718-724, 2015.

[13] M. Rakhra, R. Singh, T. K. Lohani, and M. Shabaz, "Metaheuristic and machine learning-based smart engine for renting and sharing of agriculture equipment," Mathematical Problems in Engineering, vol. 2021, Article ID 5561065, 13 pages, 2021.

[14] A. Arab and M. M. Fateh, "An uncertainty compensator for robust control of wheeled mobile robots," Advanced Robotics, vol. 29, no. 20, pp. 1303-1313, 2015.

[15] K. Shojaei, "Saturated output feedback control of uncertain nonholonomic wheeled mobile robots," Robotica, vol. 33, no. 1, pp. 87-105, 2015.

[16] S. Roy, S. Nandy, R. Ray, and S. N. Shome, "Robust path tracking control of nonholonomic wheeled mobile robot: experimental validation," International Journal of Control, Automation and Systems, vol. 13, no. 4, pp. 897-905, 2015.

[17] Q. Yao, M. Shabaz, T. K. Lohani, B. M. Wasim, G. S. Panesar, and R. K. Singh, "3D modelling and visualization for visionbased vibration signal processing and measurement," Journal of Intelligent Systems, vol. 30, no. 1, pp. 541-553, 2021.

[18] S. J. Yoo and B. S. Park, "Connectivity-preserving approach for distributed adaptive synchronized tracking of networked uncertain nonholonomic mobile robots," IEEE Transactions on Cybernetics, vol. 48, no. 9, pp. 2598-2608, 2018.

[19] V. Jagota, A. P. S. Sethi, and K. Kumar, "Finite element method: an overview," Walailak Journal of Science \& Technology, vol. 10, no. 1, pp. 1-8, 2013.
[20] A. Sgorbissa, "Integrated robot planning, path following, and obstacle avoidance in two and three dimensions: wheeled robots, underwater vehicles, and multicopters," The International Journal of Robotics Research, vol. 38, no. 7, pp. 853876, 2019.

[21] A. Kumar, V. Jagota, R. Q. Shawl et al., "Wire EDM process parameter optimization for D2 steel," Materials Today: Proceedings, vol. 37, no. 2, pp. 2478-2482, 2021.

[22] S. Dogru and L. Marques, "A physics-based power model for skid-steered wheeled mobile robots," IEEE Transactions on Robotics, vol. 34, no. 2, pp. 421-433, 2018.

[23] P. B. Dash, B. Naik, J. Nayak, and S. Vimal, "Deep belief network-based probabilistic generative model for detection of robotic manipulator failure execution," Soft Computing, 2021.

[24] G. Dhiman and A. Kaur, "HKn-RVEA: a novel many-objective evolutionary algorithm for car side impact bar crashworthiness problem," International Journal of Vehicle Design, vol. 80, no. 2/3/4, pp. 257-284, 2019.

[25] K. Albert, K. S. Phogat, F. Anhalt, R. N. Banavar, D. Chatterjee, and B. Lohmann, "Structure-preserving constrained optimal trajectory planning of a wheeled inverted pendulum," IEEE Transactions on Robotics, vol. 36, no. 3, pp. 910-923, 2020.

[26] Y. A. Kapitanyuk, A. V. Proskurnikov, and M. Cao, “A guiding vector-field algorithm for path-following control of nonholonomic mobile robots," IEEE Transactions on Control Systems Technology, vol. 26, no. 4, pp. 1372-1385, 2018.

[27] T. Yona and Y. Or, "The wheeled three-link snake model: singularities in nonholonomic constraints and stick-slip hybrid dynamics induced by Coulomb friction," Nonlinear Dynamics, vol. 95, no. 3, pp. 2307-2324, 2019.

[28] L. Ding, L. Huang, S. Li et al., "Definition and application of variable resistance coefficient for wheeled mobile robots on deformable terrain," IEEE Transactions on Robotics, vol. 36, no. 3, pp. 894-909, 2020.

[29] J. Alonso-Mora, P. Beardsley, and R. Siegwart, "Cooperative collision avoidance for nonholonomic robots," IEEE Transactions on Robotics, vol. 34, no. 2, pp. 404-420, 2018. 